\title{
The Application of Improved Sparrow Search Algorithm in Sensor Networks Coverage Optimization of Bridge Monitoring
}

\author{
Yueqi PENG, Yunqing LIU ${ }^{1}$ and Qi LI \\ Changchun University of Science and Technology, Changchun, China
}

\begin{abstract}
Aiming at the uneven random coverage distribution of wireless sensor networks sensor nodes, the sparrow search algorithm (SSA) is used to optimize the node coverage of wireless sensor networks. To improve the global search capability of SSA, the algorithm is improved and applied to the wireless sensor networks of bridge monitoring. For enhancing the coverage of the wireless sensor networks, this article uses two improved methods. One is to use the good point set theory to make the initial population evenly distributed; another is to introduce a weight factor to speed up its convergence. The experiments have proved the reliability and rationality of the algorithm. The improved algorithm is superior to other metaheuristic algorithms and provides a new idea for optimizing bridge monitoring wireless sensor networks coverage.
\end{abstract}

Keywords. sparrow search algorithm, wireless sensor networks, bridge monitoring, coverage

\section{Introduction}

Bridges play an essential role in the transportation industry. The safety of bridge construction is directly related to the security of people's lives and property. Monitoring bridges have significant practical value. Wireless sensor networks are widely used in various fields such as national defense and military, space exploration, medical and health, traffic management, smart home, etc. Wireless sensor networks have been widely used in bridge monitoring due to their convenient installation, flexible deployment, and low maintenance costs. Coverage is an essential criterion for measuring wireless sensor networks.

The coverage optimization of wireless sensor networks is a multi-objective optimization problem. A bridge monitoring system requires an appropriate deployment of monitoring sensors to achieve good monitoring results. At present, many scholars at home and abroad have conducted in-depth research on the coverage optimization of wireless sensor networks. The key to the problem is to optimize the deployment of sensor nodes in the area by adopting appropriate optimization strategies for different monitoring situations, so as to ensure sensor coverage. With the rise of meta-heuristic algorithms,

${ }^{1}$ Corresponding Author: Yunqing Liu, Changchun University of Science and Technology, Changchun, China; E-mail: mzliuyunqing@163.com. 
the use of meta-heuristic algorithms to optimize the coverage of wireless sensor networks has attracted the attention of many researchers. Literature [1] proposed a particle swarm optimization algorithm with dynamic weight optimization to uniformly cover sensor network nodes. Literature [2] proved the feasibility of the improved artificial fish swarm algorithm to optimize the coverage of wireless sensor network nodes. Literature [3] proposed an improved cuckoo search algorithm for wireless sensor networks coverage multi-objective optimization. Literature [4] used the improved glowworm swarm optimization algorithm to optimize the coverage of wireless sensor networks. Literature [5] applied a genetic algorithm to offshore wireless sensor networks. Literature [6] combined the improved heuristic ant colony algorithm with chaos operator to optimize wireless sensor networks' layout. Literature [7] designed a dichotomous fruit fly optimization algorithm applied in sensor deployment to improve senso networks coverage.

This paper uses the improved algorithm to deploy the nodes of the wireless sensor networks. By updating the position of the node, the optimal solution for each iteration is quickly calculated and the convergence speed is improved. This paper takes into account the complex and changeable environment of the bridge. It uses the characteristics of good mobility of sensor nodes, takes coverage as the final optimization goal, and combines neighbor node information to achieve uniform deployment of sensor nodes to ensure maximum coverage and high deployment reliability. The simulation experiment results show that the improved SSA has a significant improvement in the coverage optimization problem of bridge monitoring wireless sensor networks, which verifies the effectiveness of the improved algorithm.

\section{Bridge Monitoring Sensor Coverage Model}

In the deployment of bridge monitoring sensor networks, the number of sensors is often increased to increase the coverage of the sensor network. However, too many sensors deployed will generate a large number of redundant nodes, leading to data conflicts, waste of resources, and affecting network stability. Therefore, when deploying sensor networks, both network coverage and the number of nodes must be considered. This paper uses the sparrow search algorithm (SSA) in the two-dimensional plane of the bridge monitoring area.

Initialize the model, assuming that each sensor node has the same sensing radius and communication radius. Let $s=\left\{s_{1}, s_{2}, s_{3} \cdots s_{n}\right\}$ be a set of sensor nodes. The coordinate of any sensor node $s_{i}$ in the set is $\left(x_{i}, y_{i}\right)$, and the coordinate of any sensor node $s_{j}$ in the set is $\left(x_{j}, y_{j}\right)$. Then the distance between node $s_{i}\left(x_{i}, y_{i}\right)$ and node $s_{j}\left(x_{j}, y_{j}\right)$ can be obtained by the Euclidean distance calculation formula:

$$
d\left(s_{i}, s_{j}\right)=\sqrt{\left(x_{i}-x_{j}\right)^{2}+\left(y_{i}-y_{j}\right)^{2}}
$$

Discretize the monitoring area $A$ into $m \times n$ grid points. With $s_{i}\left(x_{i}, y_{i}\right)$ as the center of the circle, and $r_{i}$ as the sensor's sensing range radius. The coordinate of point 
$K$ is $\left(x_{k}, y_{k}\right)$, and the probability that the sensor node $s_{i}$ covers point $K$ is $P\left(K, s_{i}\right)$. From the Boolean perception model:

$$
P\left(K, s_{i}\right)= \begin{cases}1 & , d\left(s_{i}, s_{j}\right) \leq r_{i} \\ 0 & , d\left(s_{i}, s_{j}\right)>r_{i}\end{cases}
$$

For any coordinate point $(x, y)$, as long as there is a sensor node in the node set $\mathrm{C}$ such that $P_{\text {cov }}\left(x, y, c_{i}\right)=1$. The joint measurement coverage $P_{\text {cov }}(C)$ of the sensor node set is:

$$
P_{\text {cov }}(C)=1-\prod_{c_{i} \in C}\left(1-P_{\text {cov }}\left(x, y, c_{i}\right)\right)
$$

The wireless sensor network coverage rate can be expressed as the ratio of the total monitoring coverage rate to the monitoring area:

$$
P_{\text {area }}=\frac{\sum_{x=1}^{m} \sum_{y=1}^{n} P_{\text {cov }}(C)}{m \times n}
$$

\section{Sparrow Search Algorithm}

Xue and Shen [8] propose the SSA. It is inspired by the foraging and anti-predation behavior of sparrows. It has the characteristics of fast convergence, strong stability and good robustness. This algorithm can solve practical problems such as nonlinear optimization and reducer design, and has broad development prospects.

The algorithm divides sparrows into two different types through different foraging strategies, namely producers and scroungers. Producers are responsible for finding food sources, and scroungers obtain food through producers. Sparrows with abundant food resources are more vulnerable to attack. Sparrow's choice of foraging strategy plays an important role in the individual's energy reserve, while most sparrows have low energy reserves. Sparrows on the outer edge of the group are more vulnerable to attack and gather in the middle. Sparrows in the middle of the group will approach their neighbors to reduce the probability of encountering danger. When the sparrow finds danger, it will scream and the whole sparrow flock will fly away.

Assuming a sparrow is $x$, in the search process, producers with high adaptability are given priority to get food. The producers are responsible for finding food and guiding the migration of the entire group. The position of producers is updated as follow:

$$
X_{i, j}^{t+1}= \begin{cases}X_{i j}^{t} \cdot \exp \left(\frac{-i}{\alpha \cdot \text { iter }_{\max }}\right) & \text {,if } R_{2}<S T \\ X_{i, j}^{t}+Q \cdot L & \text {,if } R_{2} \geq S T\end{cases}
$$


Where $t$ is the current iteration. The value of $j$ is $1,2 \cdots d \cdot X_{i, j}^{t}$ is the value of the $j$ th dimension during the iteration of the $i$ th sparrow. iter $_{\max }$ is the maximum number of iterations. $\alpha$ is a random number in $(0,1] \cdot R_{2} \in[0,1]$ is the alarm value, and $S T \in[0.5,1.0]$ is the safety threshold. $Q$ is a random number that obeys normal distribution. $L$ is a $1 \times d$ matrix, and each element in the matrix is 1 .

When $R_{2}<S T$, it means that there is no danger around, and the producers perform a global search. When $R_{2} \geq S T$, it means that sparrows have found a danger, and the entire sparrow group is evacuated to a safe area.

When the scroungers found that the producers had found the food, they immediately got the food. If the scroungers do not get food, they follow the producers to find food. The position update formula for scroungers is expressed as follows:

$$
X_{i, j}^{t+1}= \begin{cases}Q \cdot \exp \left(\frac{X_{\text {worst }}^{t}-X_{i, j}^{t}}{i^{2}}\right) & \text {,if } i>n / 2 \\ X_{p}^{t+1}+\left|X_{i, j}^{t}-X_{p}^{t+1}\right| \cdot A^{+} \cdot L & \text {,otherwise }\end{cases}
$$

Where $X_{P}$ is the best position occupied by the producer, and $X_{\text {worst }}$ is the worst position in the current global situation. $A$ is a $1 \times d$ matrix, each element in the matrix is randomly assigned as 1 or -1 , satisfying $A^{+}=A^{T}\left(A A^{T}\right)^{-1}$. When $i>n / 2$, it indicates that the $i$ th scrounger has poor adaptability and may starve to death.

Assume that sparrows that perceive danger account for $10 \%$ to $20 \%$ of the total number of sparrows. The initial positions of these sparrows are randomly generated in the population. The mathematical model can be expressed as follows:

$$
X_{i, j}^{t+1}= \begin{cases}X_{\mathrm{best}}^{t}+\beta \cdot\left|X_{i, j}^{t}-X_{\mathrm{best}}^{t}\right| & \text {,if } f_{i}>f_{g} \\ X_{i, j}^{t}+K \cdot\left(\frac{\left|X_{i, j}^{t}-X_{\text {worst }}^{t}\right|}{\left(f_{i}-f_{W}\right)+\varepsilon}\right) & \text {,if } f_{i}=f_{g}\end{cases}
$$

Where $X_{\text {best }}$ is the best position in the current global situation. $\beta$ is the parameter that controls the step length. $K \in[-1,1]$ is a random number. $f_{i}$ is the fitness value of the current sparrow. $f_{g}$ is the current global best the fitness value. $f_{w}$ is the current global worst fitness value. $\varepsilon$ is the minimum constant to avoid zero-division-error.

When $f_{i}>f_{g}$, it means that the sparrows are at the edge of the group and are safe. When $f_{i}=f_{g}$, it means that the sparrows in the group are aware of the danger. $K$ is the direction of the sparrow's movement, which is the parameters control step length. 


\section{Improved Sparrow Search Algorithm}

In order to design a reasonable initial population of SSA, it is necessary to consider how to use as few individuals as possible to comprehensively characterize the distribution of all potential solutions in the entire space. If the initial population is randomly generated, it is difficult to find a solution to the problem. The good point set theory is used to improve the algorithm for population initialization so as to solve the problem of uneven population distribution. The good point set theory was first proposed by Hua Luogeng [9]. The good point set theory selects points with less deviation than randomly selected points, and its deviation is equivalent to the square root level of random selection [10].

The basic definition and structure of the good point set theory are as follows: Let $G_{S}$ be the unit cube of $S$ dimensional Euclidean space, that is $x \in G_{S}$, $x=\left(x_{1}, x_{2}, x_{3}, \cdots x_{S}\right)$. Where $0 \leq x_{i} \leq 1, i=1,2, \ldots s$, the point $r=\left(r_{1}, r_{2}, \ldots, r_{S}\right)$ in $G_{S}$, let $r \in G_{S}$, then:

$$
P_{n}(k)=\left\{\left(\left\{r_{1} k\right\}, \ldots,\left\{r_{s} k\right\}\right), k=1,2, \ldots, n\right\}
$$

$P_{n}(k)$ is a good point set, and $r$ is a good point. The deviation $\varphi(n)$ of the point set satisfies:

$$
\varphi(n)=C(r, \varepsilon) n^{-1+\varepsilon}
$$

where $C(r, \varepsilon)$ is a constant related only to $r, \varepsilon$ ( $\varepsilon$ is any positive number), take $r k=2 \cos (2 \pi k / p), 1 \leq k \leq D \cdot p$ is the smallest prime of satisfied $(p-D) / 2 \geq D$.

When generating the initial population, in the case of the same number of points, the points selected by the good point set are more uniform than the points randomly selected. Therefore, mapping the best points of $G_{S}$ to the target solution space makes the initial population more ergodic, effectively avoiding the algorithm from falling into the local optimal value and stagnating, and improving the global convergence.

As the number of algorithm iterations continues to increase, the algorithm's optimization capability gradually tends to converge, and once it converges to the local optimum, it is difficult to jump out. Adding a weight factor to the algorithm can effectively improve the ability of the algorithm to jump out of the local optimal solution. This paper uses the weight factor to improve the original algorithm. The weight factor calculation formula is:

$$
\omega=2-t \cdot \frac{2}{i t e r_{\max }}
$$

Where $t$ is the number of iterations. iter $_{\text {max }}$ is the maximum number of iterations. 


\section{Simulation and Experiment Analysis}

Aiming at the problem of low coverage of the random deployment of sensors for bridge monitoring, the improved SSA is used to optimize the deployment of sensor nodes. Based on the wireless sensor network's sensor node coverage optimization strategy for simulation, Matlab 2018b programming is selected to implement the sensor node coverage optimization program, and the environmental parameters are listed in Table 1.

Table 1. Set the same environmental parameters for the three algorithms

\begin{tabular}{cc}
\hline parameters & value \\
\hline monitoring area & $100 \mathrm{~m} \times 100 \mathrm{~m}$ \\
number of nodes & 25 \\
communication radius & $10 \mathrm{~m}$ \\
population size & 25 \\
maximum number of iterations & 1000 \\
\hline
\end{tabular}

To further compare the performance of the algorithm, this paper compares the particle swarm algorithm, the SSA, and the improved SSA. The coverage rate of sensor nodes is optimized by the particle swarm algorithm, and the coverage rate is relatively low, only $62.3 \%$. The sensor nodes optimized by the original SSA are evenly distributed, with a coverage rate of $74.6 \%$. The improved SSA algorithm has a more uniform sensor coverage, reduces the overlap of sensors, and the coverage rate is $77.1 \%$, thereby optimizing the deployment of bridge monitoring. Sensor nodes can effectively improve sensor performance. The sensor node coverage optimization is shown in Figure 1.

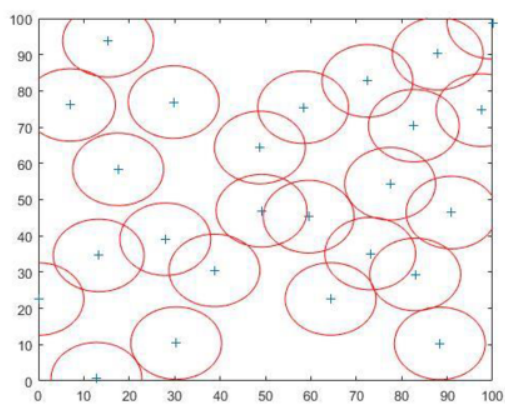

(a) particle swarm optimization

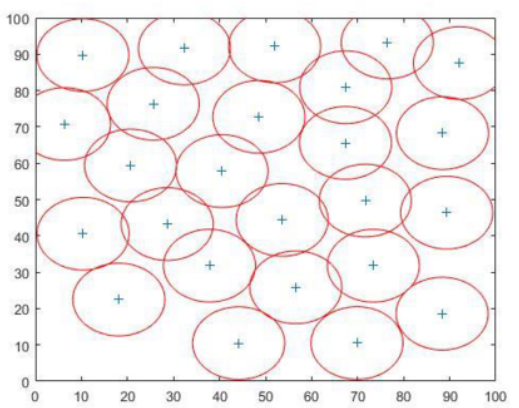

(b) sparrow search algorithm

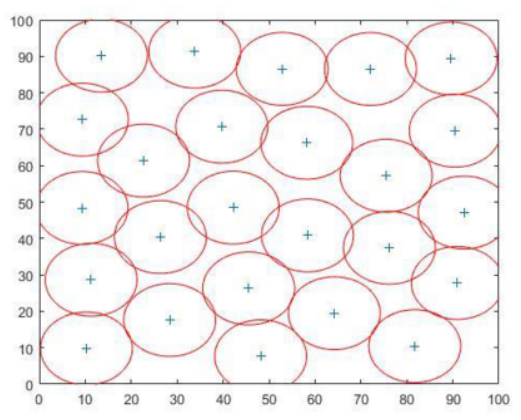

(c) improved sparrow search algorithm

Figure 1. Sensor node coverage optimization 
Figure 2 is an experimental comparison diagram of the particle swarm optimization, the original SSA and the improved SSA in this paper. The experiment deployed the same number of sensor nodes in the same monitoring area. Taking into account the interference of randomness on the experimental results, the three algorithms were tested ten times, and the average coverage rate was taken. It can be seen from Figure 2 that under the same experimental conditions, the improved SSA in this paper can effectively improve the node coverage of wireless sensor networks.

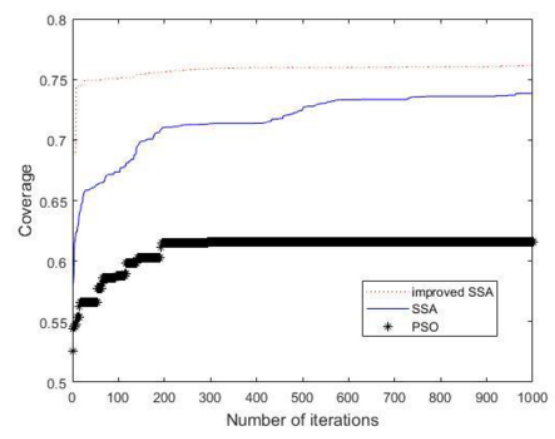

Figure 2. Comparison of coverage.

\section{Conclusion}

As a new algorithm, SSA has fewer parameters, high search efficiency, stable optimization results, and strong robustness, which provides an effective way for bridge monitoring sensor coverage. The original SSA has defects such as slow convergence speed and insufficient population vitality in the evolution process. This paper proposes an improved SSA and applies it to the problem of bridge monitoring sensor coverage optimization. The experiment verified the feasibility and effectiveness of SSA. In addition, the improved strategy proposed in this paper can further improve the search performance of the algorithm. The improved SSA search performance is higher and the optimization result is more stable.

\section{References}

[1] Xu Yiming, Peng Yong, Zheng Chuhong, Liao Yi, Node energy balanced coverage strategy in WSNs based on improved PSO algorithm[J], Transducer and Microsystem Technologies 39 (2020), 29-32.

[2] Huang Yuyue, Li Keqing, Coverage optimization of wireless sensor networks based on artificial fish swarm algorithm[J], Application Research of Computers, 30 (2013), 554-556.

[3] Pan Hao, Fui Hua, Wireless sensor coverage multi-objective optimization improved based on cuckoo search algorithm[J], Journal of Jilin Normal University (Natural Science Edition) 38 (2017), 125-129.

[4] Liu Zhouzhou, Wang Fubao, Zhang Kewang, Performance analysis of improved glowworm swarm optimization algorithm and the application in coverage optimization of WSNs[J], Chinese Journal of Sensors and Actuators 26 (2013), 675-682.

[5] Zhang Guihong. Application of genetic algorithm in optimal configuration of maritime wireless sensors[J], Ship Science and Technology 41 (2019), 148-150.

[6] Duan Yujun, Wang Yaoli, Chang Qing, Liu Xin, Wireless sensor deployment optimization based on improved IHACA-CpSPIEL algorithm [J], Journal of Computer Applications 40 (2020), 793-798.

[7] Jia Zhe, Novel dichotomy fruit fly optimization algorithm in sensor deployment[J], Microcontrollers and Embedded Systems 19 (2019), 36-39. 
[8] Xue Jiankai, Shen Bo, A novel swarm intelligence optimization approach: sparrow search algorithm[J], Systems Science and Control Engineering 8 (2020), 22-34.

[9] Hua Luogeng, Wang Yuan, Application of number theory in approximate analysis [M], Science Press, Beijing, 1978.

[10] Fang Xian, Tie Zhixin, Li Jingming, et al, A parallel search good-point set glowworm swarm optimization of re-created population after clustering of multi-modal functions[J], Journal of Zhejiang Sci-Tech University (Natural Sciences) 37 (2017), 843-850. 